\title{
Realization of a continuous-wave single-frequency tunable Nd:CYA laser
}

\author{
Huiqi Yang (杨慧琦) ${ }^{1}$, Pixian Jin (靳丕铦 $)^{1,2}$, Jing Su (苏 静) ${ }^{1,2}$ ， Xiaodong Xu (徐晓东) $)^{3}$, Jun Xu (徐 军) $)^{4}$, and \\ Huadong Lu (卢华东) $)^{1,2^{*}}$ \\ ${ }^{1}$ State Key Laboratory of Quantum Optics and Quantum Optics Devices, Institute of Opto-electronics, Shanxi University, Taiyuan 030006, China \\ ${ }^{2}$ Collaborative Innovation Center of Extreme Optics, Shanxi University, Taiyuan 030006, China \\ 3 Jiangsu Key Laboratory of Advanced Laser Materials and Devices, School of Physics and Electronic Engineering, Jiangsu Normal University, \\ Xuzhou 221116, China \\ ${ }^{4}$ School of Physics Science and Engineering, Institute for Advanced Study, Tongji University, Shanghai 200092, China
}

*Corresponding author: luhuadong@sxu.edu.cn

Received November 4, 2021 | Accepted December 13, 2021 | Posted Online January 12, २०२2

\begin{abstract}
We demonstrate an all-solid-state continuous-wave (CW) single-frequency tunable $1.08 \mu \mathrm{m}$ laser, which is realized by

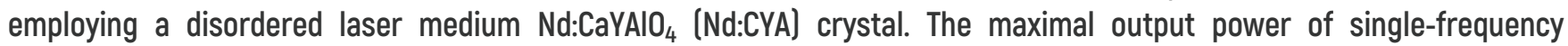
$1.08 \mu \mathrm{m}$ laser is $1 \mathrm{~W}$. By rotating the incident angle of the intracavity etalon (IE), the maximal tuning range of $183.71 \mathrm{GHz}$ is achieved. After the IE is locked to the oscillating longitudinal mode of the laser, the continuous tuning range of $60.72 \mathrm{GHz}$ for $1.08 \mu \mathrm{m}$ laser is achieved by scanning the cavity length. To the best of our knowledge, this is the first demonstration of a CW single-frequency widely tunable $1.08 \mu \mathrm{m}$ laser based on $\mathrm{Nd}$ :CYA crystal.
\end{abstract}

Keywords: all-solid state laser; continuous wave; continuous tuning; $\mathrm{Nd}: \mathrm{CaYAlO}_{4}$ crystal.

DOI: 10.3788/COL202220.031403

\section{Introduction}

All-solid-state continuous-wave (CW) single-frequency lasers have been established as efficient devices, capable of generating high stability, low intensity noise, and perfect beam quality ${ }^{[1]}$. Among those lasers with diverse output wavelengths, the allsolid-state single-frequency lasers with the operating wavelength of $1.08 \mu \mathrm{m}$ as promising light sources have been demonstrated to directly generate the continuous variable non-classical light fields by only utilizing one $a$-cut type-II non-critical phasematching $\mathrm{KTiOPO}_{4}$ (KTP) crystal ${ }^{[2-6]}$. The achieved nonclassical light fields act as the basic resources of the quantum computing (QC) and quantum information network (QIN). Therefore, the single-frequency CW $1.08 \mu \mathrm{m}$ lasers can be broadly applied in quantum optics and quantum technologies. Moreover, because the wavelength of $1.08 \mu \mathrm{m}$ corresponds to the cesium $6 \mathrm{~S} \rightarrow 7 \mathrm{~S}$ two-photon transition ${ }^{[7]}$, the singlefrequency $1.08 \mu \mathrm{m}$ lasers can also be applied in atomic physics. In order to precisely match the cesium $6 \mathrm{~S} \rightarrow 7 \mathrm{~S}$ two-photon transition line, it is required to realize the frequency tuning of the single-frequency CW $1.08 \mu \mathrm{m}$ lasers. In recent years, the $\mathrm{Nd}: \mathrm{CaYAlO}_{4}$ (Nd:CYA) crystal as a candidate to emit the wavelength of the $1.08 \mu \mathrm{m}$ laser has become more and more popular. In addition, several intrinsic advantages of the Nd:CYA crystal make it suitable for being used to generate the $1.08 \mu \mathrm{m}$ lasers. Firstly, its absorption peak falls just around $807 \mathrm{~nm}$ $\left({ }^{4} \mathrm{I}_{9 / 2} \rightarrow{ }^{4} \mathrm{~F}_{5 / 2}+{ }^{2} \mathrm{~F}_{9 / 2}\right){ }^{[8]}$, which corresponds to the wavelength of the most mature laser diode (LD). Then, benefiting greatly from the disordered crystal structure ${ }^{[9,10]}$, the Nd:CYA crystal has large inhomogeneous absorption (full width at halfmaximum is $5 \mathrm{~nm}$ ) and emission broadened spectra (full width at half-maximum is $20 \mathrm{~nm}$ ). The spectral properties of the $\mathrm{Nd}$ :CYA crystal makes it a good candidate to attain tunable and ultrafast lasers ${ }^{[8]}$. Owing to these desirable characteristics, a large number of $\mathrm{CW}$ transverse electromagnetic $\left(\mathrm{TEM}_{00}\right)$ mode lasers and tunable $Q$-switched lasers based on the Nd:CYA crystal have been studied. In 1989, Haracio et al. firstly, to the best of our knowledge, presented the laser action of $\mathrm{Nd}$ :CYA crystal and proved that CYA was a good host for rare-earth ions, and the maximum output power of the CW $\mathrm{TEM}_{00}$ mode at $1.08 \mu \mathrm{m}$ was about $80 \mathrm{~mW}^{[9]}$. Stephens et al. further showed the continuous tunable single-mode laser of the Nd:CYA crystal from 1077.5 to $1084.5 \mathrm{~nm}$ with a Lyot filter in the resonator and the maximum output power with only $15 \mathrm{~mW}$ in $1992^{[10]}$, which further confirmed that the emission spectra were broadened enough to be used for tuning and optically pumping metastable helium atoms. Yu et al. demonstrated a 5.16 W CW $1.08 \mu \mathrm{m}$ laser with a Nd:CYA crystal and further obtained a passively $Q$-switched laser with a Cr:YAG in the 
cavity in $2010^{[11]}$. In 2011, Fu et al. reported a CW frequencydoubled green laser at $540 \mathrm{~nm}$ in KTP crystal in a type-II phasematching direction performed with a diode pumped Nd:CYA laser. The output power of $540 \mathrm{~nm}$ laser reached $324 \mathrm{~mW}$, and its beam quality was 1.34 and 1.22 when the pump power was $18.2 \mathrm{~W}^{[12]}$. Because of the multi-mode operation, there was cross saturation in the Nd:CYA crystal, sum frequency in the KTP crystal, and large fluctuation of the output power. However, up to now, there is no report on a stable CW single-longitudinal-mode (SLM) tunable laser based on Nd:CYA crystal, to the best of our knowledge. In this paper, we first, to the best of our knowledge, report a CW single-frequency tunable CW Nd:CYA laser with good performance. The output power of the single-frequency $1.08 \mu \mathrm{m}$ laser reaches up to $1 \mathrm{~W}$. The maximal and continuous tuning ranges of the $1.08 \mu \mathrm{m}$ laser are up to $183.71 \mathrm{GHz}$ and $60.72 \mathrm{GHz}$, respectively. The results prove that it can be an excellent source for QIN and QC.

\section{Experimental Design}

The configuration of the designed CW single-frequency tunable $1.08 \mu \mathrm{m}$ laser system is shown in Fig. 1. The figure-eight-shaped ring cavity with the length of $283.84 \mathrm{~mm}$ is formed by two plane mirrors $M_{1}, M_{2}$ and two plane-concave mirrors $M_{3}, M_{4}$. The curvature radii of both concave mirrors are $50 \mathrm{~mm}$. The input coupler $\mathrm{M}_{1}$ is coated with high transmission (HT) film at $808 \mathrm{~nm}\left(T_{808}>95 \%\right)$ and high reflection (HR) film at $1.08 \mu \mathrm{m}\left(R_{1080}>99.5 \%\right) . \mathrm{M}_{2}$ is coated with partial transmission film $\left(T_{1080}=4 \%\right) . \mathrm{M}_{3}$ is coated with $\mathrm{HR}$ film at $1.08 \mu \mathrm{m}$ $\left(R_{1080}>99.5 \%\right) . \mathrm{M}_{4}$ is a dichroic mirror coated with HR film at $1.08 \mu \mathrm{m}\left(R_{1080}>99.5 \%\right)$ and HT film at $540 \mathrm{~nm}\left(T_{540}>95 \%\right)$. The pump source is a fiber coupled LD with the output power of $15 \mathrm{~W}$ at $808 \mathrm{~nm}$ (Dilas). Through scanning the LD's temperature, its wavelength could be precisely tuned to the maximum absorption peak of the Nd:CYA crystal. The core diameter and numerical aperture (N. A.) of the fiber are $400 \mu \mathrm{m}$ and 0.22 , respectively. A telescope system, consisting of two lenses $f_{1}$ and $f_{2}$ with focal lengths of $30 \mathrm{~mm}$ and $50 \mathrm{~mm}$,

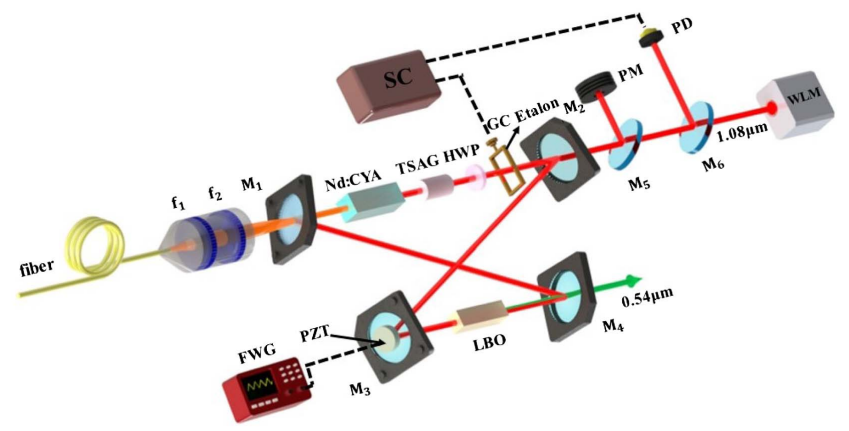

Fig. 1. Schematic diagram of the LD-pumped CW SLM tunable dualwavelength $1.08 \mu \mathrm{m}$ and $0.54 \mu \mathrm{m}$ laser. HWP, half-wave-plate; GC, galvanometer scanner; SC, servo controller; PM, power meter; PD, photodetector; WLM, wavelength meter; FWG, function waveform generator; PZT, piezoelectric transducer. respectively, is designed to focus the pump light at the center of the gain medium. The gain medium is an $a$-cut $\mathrm{Nd}$ :CYA crystal with the dimensions and $\mathrm{Nd}$-doping concentration of $3 \mathrm{~mm} \times 3 \mathrm{~mm} \times 6 \mathrm{~mm}$ and $1 \%$ (atomic fraction), respectively. It is wrapped with indium foil in a copper oven to realize timely temperature adjustment. The temperature of the $\mathrm{Nd}$ :CYA crystal is set as $27.79^{\circ} \mathrm{C}$. Both ends of the crystal are coated with antireflection (AR) films at both $808 \mathrm{~nm}$ and $1.08 \mu \mathrm{m}$. The optical diode (OD), composed by a terbium scandium aluminum garnet (TSAG) crystal with the size of $\phi 4 \mathrm{~mm} \times 4.5 \mathrm{~mm}$ surrounded by permanent magnet and a half-wave plate (HWP), is inserted into the resonator to ensure the unidirectional operation of the laser. Compared to a terbium gallium garnet (TGG) magneto-optic crystal, the TSAG crystal plays a greater role in better magnetooptical rotation. A type-I phase-matching lithium triborate (LBO) crystal $^{[13]}$ with the dimensions of $3 \mathrm{~mm} \times 3 \mathrm{~mm} \times$ $15 \mathrm{~mm}$ is placed at the beam waist between the mirrors $M_{3}$ and $\mathrm{M}_{4}$ to introduce sufficient nonlinear loss to efficiently suppress the multi-mode oscillation and mode-hopping phenomenon ${ }^{[14,15]}$. The operating temperature of $\mathrm{LBO}$ is set as $136.9^{\circ} \mathrm{C}$, which corresponds to the optimum phase-matching value of $1.08 \mu \mathrm{m}$ laser. In order to further realize the continuous frequency tuning of the laser, an intracavity etalon (IE) made of fused silica with the thickness of $0.5 \mathrm{~mm}$ is inserted into the cavity. A long piezoelectric transducer (PZT) is mounted on $\mathrm{M}_{3}$ to realize continuous scans of the cavity length by applying voltage to it. Most output of the $1.08 \mu \mathrm{m}$ laser from $\mathrm{M}_{2}$ is reflected by $\mathrm{M}_{5}$ to monitor and record the power of the $1.08 \mu \mathrm{m}$ laser. The leaked laser from $\mathrm{M}_{5}$ is split into two parts by $\mathrm{M}_{6}$. One part is coupled into the photodetector (PD) to lock the IE with the assistance of the homemade servo controller (SC). The other part is injected into the wavelength meter (WLMWS6, HighFinesse) to monitor the wavelength of the output laser.

It is well known that the equivalent thermal lens is a critical parameter for the cavity design of diode-pumped all-solid-state lasers $^{[16]}$. The thermal conductivities of the Nd:CYA crystal are $3.7 \mathrm{~W} /(\mathrm{m} \cdot \mathrm{K})$ (parallel to $a$ axis) and $3.3 \mathrm{~W} /(\mathrm{m} \cdot \mathrm{K}$ ) (parallel to $c$ axis $)^{[17-19]}$, and the thermo-optical coefficients are $-7.8 \times 10^{-6} \mathrm{~K}^{-1}$ (parallel to $a$ axis) and $-8.7 \times 10^{-6} \mathrm{~K}^{-1}$ (parallel to $c$ axis $)^{[20]}$. According to Ref. [20], the thermal coefficient of the optical path $[W=\mathrm{d} n / \mathrm{d} T+(n-1) \alpha]$ is measured as $1.2 \times 10^{-6} \mathrm{~K}^{-1}$ for $E \perp c$ polarization of $a$-cut Nd:CYA crystal, where $n$ is the refractive index $\left(n_{o}=1.886, n_{e}=1.909\right)$, and $\alpha$ is the thermal expansion coefficient $\left(\alpha_{a}=10.2 \mathrm{~K}^{-1}\right.$, $\left.\alpha_{c}=10.2 \mathrm{~K}^{-1}\right)$. Benefiting from the negative thermo-optic coefficients as well as the small and positive thermal coefficients of the optical path, the equivalent thermal lens of the Nd:CYA crystal is relatively weak. The calculated stability range depicted in Fig. 2 is broad enough to obtain a stable single-frequency CW $1.08 \mu \mathrm{m}$ laser. In order to obtain greater gain under the low pump power with the CW pumping mode, the waist radii of the pump laser and oscillating laser at the middle of the Nd:CYA crystal are designed as $320 \mu \mathrm{m}$ and $365 \mu \mathrm{m}$, respectively, after taking the equivalent thermal lens focal length of the Nd:CYA crystal into account. 


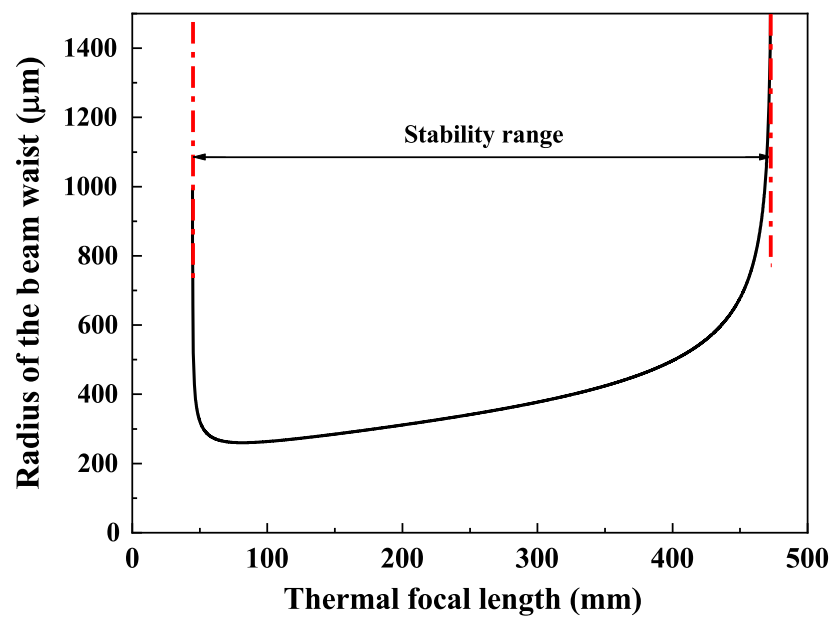

Fig. 2. Radius of the beam waist in the position of the Nd:CYA crystal versus the thermal focal length.

\section{Experimental Results and Discussion}

After precisely optimizing the laser system, the stable tunable dual-wavelength laser with good performance is obtained. The output powers of the $1.08 \mu \mathrm{m}$ and $0.54 \mu \mathrm{m}$ lasers are recorded by a power meter (PM30, Coherent Co., Ltd.), as illustrated in Fig. 3. The results show that the threshold power of the $1.08 \mu \mathrm{m}$ laser is $10.53 \mathrm{~W}$. The maximum output powers of the $1.08 \mu \mathrm{m}$ and $0.54 \mu \mathrm{m}$ lasers reach $1 \mathrm{~W}$ and $34.9 \mathrm{~mW}$, respectively, with the pump power of $13.08 \mathrm{~W}$. The slope efficiency of the $1.08 \mu \mathrm{m}$ laser is up to $38.04 \%$. The polarization degree of the output $1.08 \mu \mathrm{m}$ laser is measured by a polarization analyzer (PAX1000IR1/M, Thorlabs Co., Ltd.). The polarization ratio of the $1.08 \mu \mathrm{m}$ laser is better than $100: 1$, which is ascribed to the high gain of $E \perp c$ polarization for the $\mathrm{Nd}$ :CYA crystal. The power stability of the $1.08 \mu \mathrm{m}$ laser in $4 \mathrm{~h}$ is recorded in Fig. 4, which illustrates that the power stability is better than $\pm 0.86 \%$. The transverse-mode beam quality of the $1.08 \mu \mathrm{m}$ laser

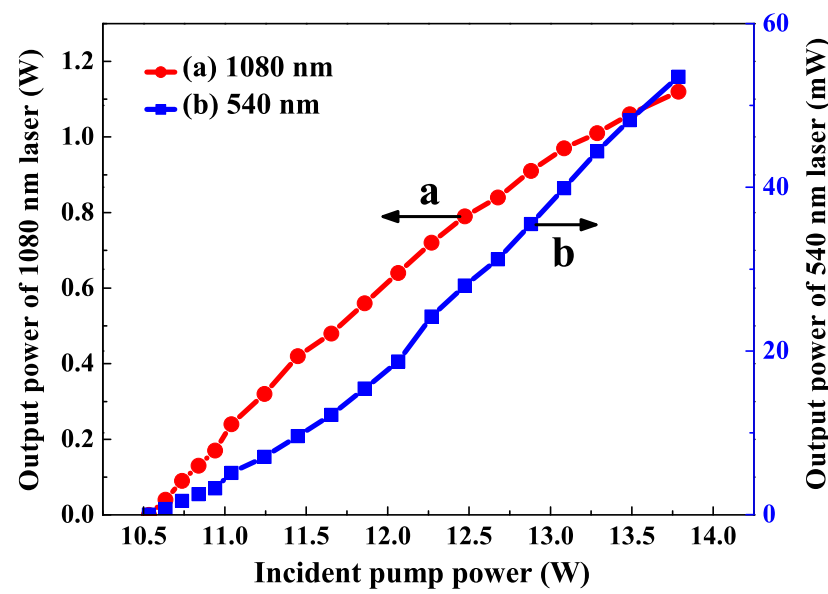

Fig. 3. Output power of $1.08 \mu \mathrm{m}$ and $0.54 \mu \mathrm{m}$ lasers as a function of the incident pump power.

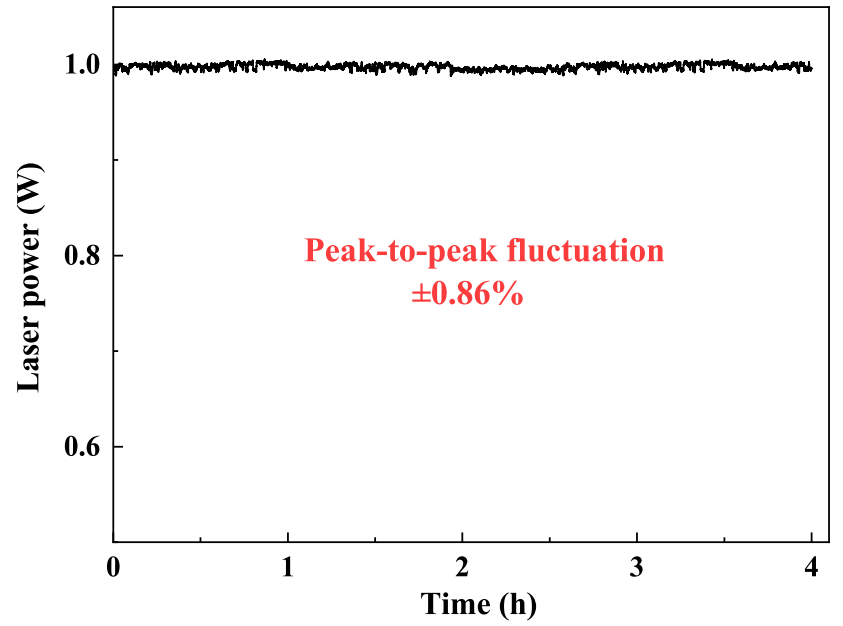

Fig. 4. Measured power stability of the $1.08 \mu \mathrm{m}$ laser in $4 \mathrm{~h}$.

is measured by employing an $M^{2}$ meter (M2SET-BP209IR/M, Thorlabs Co., Ltd.). The measured spatial beam profiles as well as corresponding caustic curves are shown in Fig. 5. The results show that the beam qualities of the $1.08 \mu \mathrm{m}$ laser are $M_{x}^{2}=1.11$ and $M_{y}^{2}=1.06$, respectively. The longitudinal-mode structure of the laser is monitored by a scanned temperature-controlled Fabry-Perot cavity (F-P-100, Yuguang Co., Ltd.). The free spectral range (FSR) and finesse of the F-P cavity are $750 \mathrm{MHz}$ and 120, respectively. From the monitored transmission curve shown in Fig. 6, it can be seen that the stable SLM operation is achieved, which benefits from the sufficient nonlinear loss introduced by the frequency-doubled crystal LBO. To the best of our knowledge, it is the first time to achieve a CW singlefrequency tunable $\mathrm{Nd}$ :CYA laser.

Combining the IE and Nd:CYA crystal with a broad-band fluorescence spectrum, the wide tuning characteristics of the CW SLM Nd:CYA laser are investigated. Through rotating the incident angle of the inserted IE by continuously varying the voltage supplied to the galvanometer scanner (GC), the

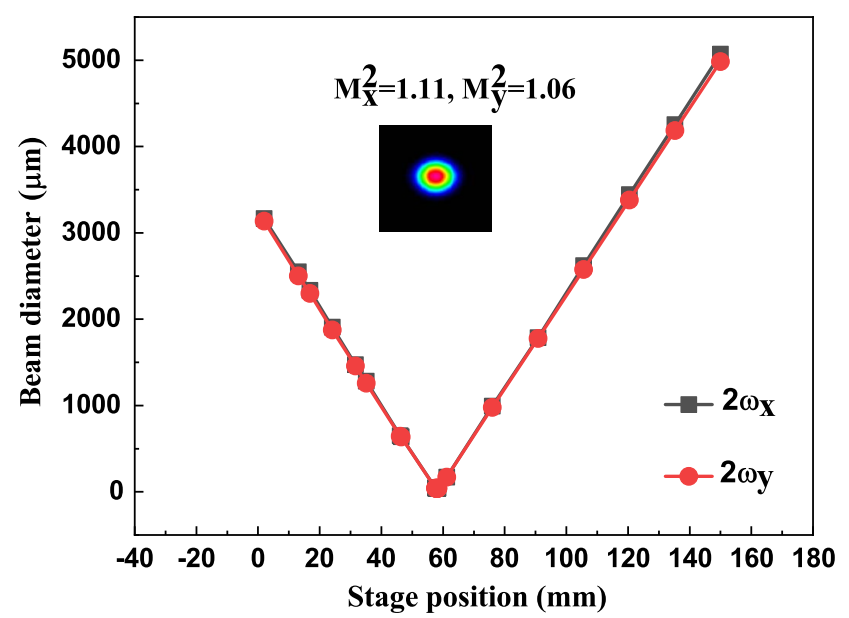

Fig. 5. Beam quality of the $1.08 \mu \mathrm{m}$ laser. 


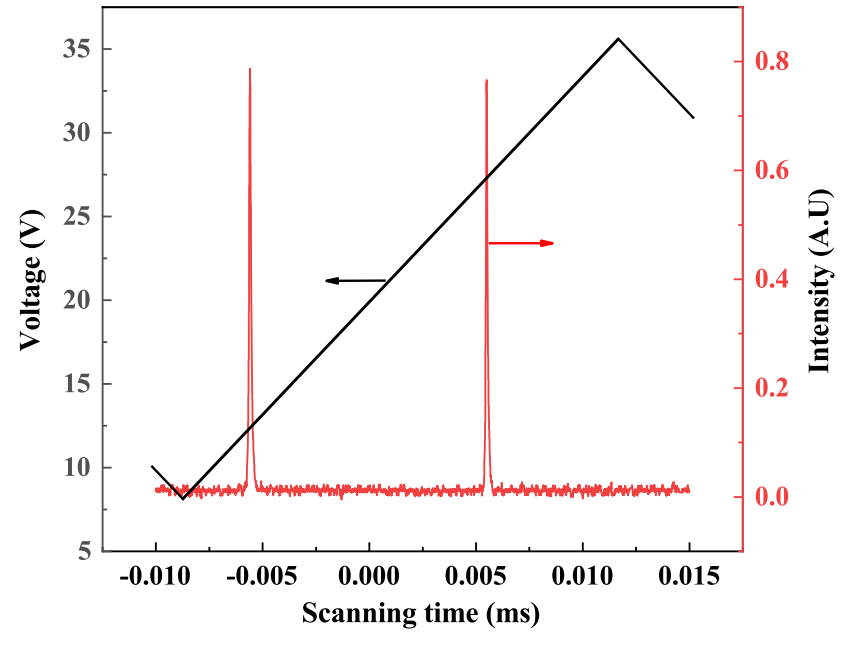

Fig. 6. Monitored longitudinal-mode structure of the $1.08 \mu \mathrm{m}$ laser.

coarse tuning ability of IE is firstly recorded by the wavelength meter in Fig. 7. When the incident angle of IE is $0^{\circ}$, the laser wavelength is $1081.4007 \mathrm{~nm}$. With the increase of the voltage supplied to the GC from $0 \mathrm{~V}$ to $1.98 \mathrm{~V}$, the wavelength discontinuously decreases from $1081.4007 \mathrm{~nm}$ to $1081.1207 \mathrm{~nm}$. In the case of $1.98 \mathrm{~V}$ supplied to the GC, the obvious wavelength-hop between $1081.1207 \mathrm{~nm}$ and $1081.8282 \mathrm{~nm}$ is observed. When further increasing the voltage from $1.98 \mathrm{~V}$ to $3.663 \mathrm{~V}$, the measured output wavelength shifts to a shorter wave from $1081.8282 \mathrm{~nm}$ to $1081.1120 \mathrm{~nm}$. The experimental results illustrate that the tuning range of the IE is $183.71 \mathrm{GHz}$, which is consistent with the FSR of the adopted IE. Further, in order to implement the continuous frequency tuning of the laser, we build a phase-lock system consisting of a photodiode (ETX 500, JDSU Corporation), function generator (FG), and homemade SC to lock the IE to the oscillating longitudinal mode of the laser ${ }^{[21,22]}$. After the transmission peak of the IE is locked to the oscillating longitudinal mode of the laser, continuous tuning with free mode-hopping is successfully realized. At this time, the

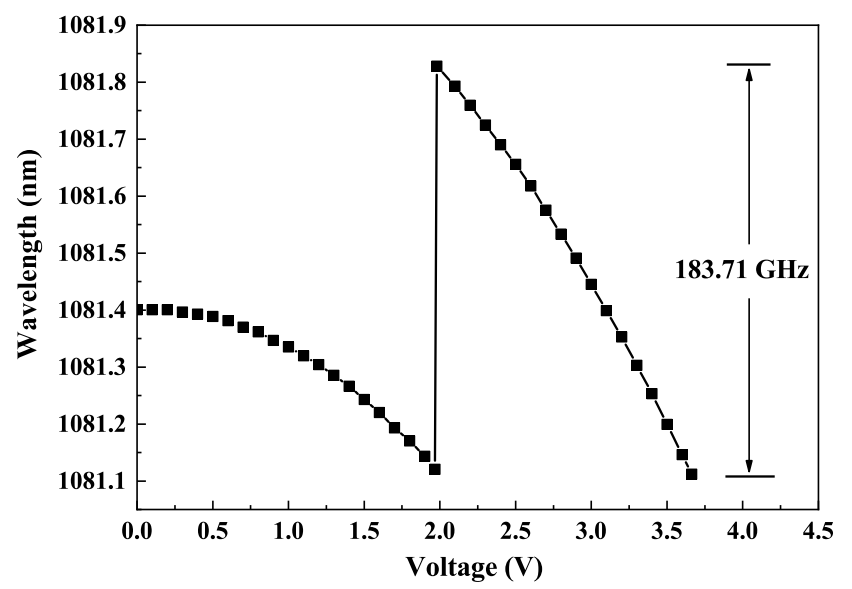

Fig. 7. The Maximum tuning range of the $1.08 \mu \mathrm{m}$ laser by rotating the incident angle of IE.

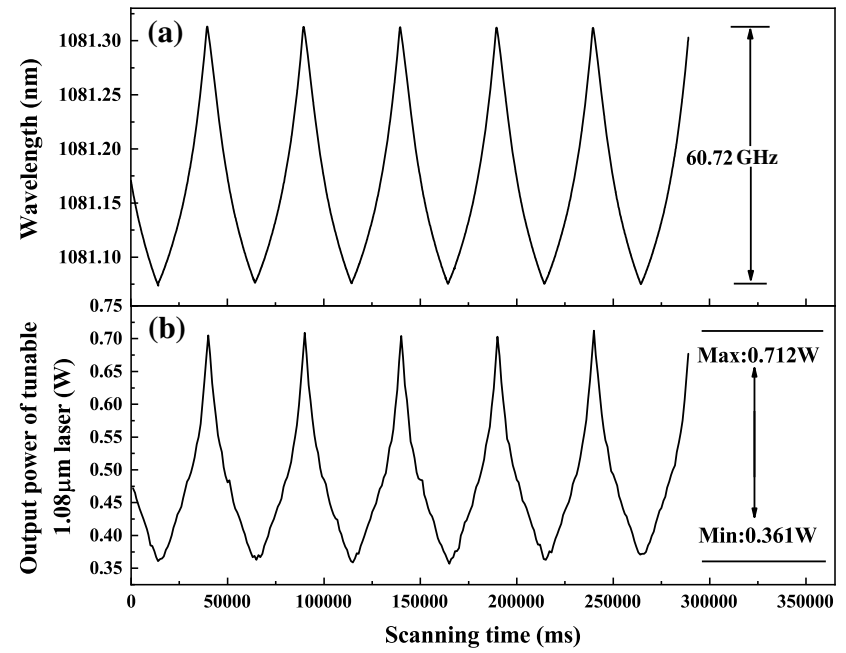

Fig. 8. Experimental results of the continuous frequency tuning. (a) Continuous frequency tuning of the $1.08 \mu \mathrm{m}$ laser versus scanning time. (b) Corresponding power variation of the $1.08 \mu \mathrm{m}$ laser in the process of continuous frequency tuning.

length of the resonator is continuously scanned by loading a voltage scanning signal with the amplitude and frequency of $150 \mathrm{~V}$ and $20 \mathrm{mHz}$, respectively, onto the PZT. As depicted in Fig. 8(a), the fine and continuous wavelength tuning range of $60.72 \mathrm{GHz}$ at $1.08 \mu \mathrm{m}$ is achieved. It can also be concluded from Fig. 8(a) that the output wavelength of the $1.08 \mu \mathrm{m}$ laser varies as nonlinearly as the scanning time increased, which results from the nonlinearity of the PZT. The highest tuning speed of output wavelengths is $3.679 \times 10^{-5} \mathrm{~nm} / \mathrm{ms}$. It is expected that the broader continuous frequency tuning range of the CW SLM Nd:CYA laser could be attained if a PZT with longer displacement is employed in the experiment. In the tuning process, a fraction of the output power of the achieved tunable singlefrequency CW $1.08 \mu \mathrm{m}$ laser at different tuning wavelengths is simultaneously monitored, and the results are shown in Fig. 8(b). The results depict that the periodic variation trends of the output power are similar to that of the continuous frequency tuning. The maximum and minimum output powers of the tunable $1.08 \mu \mathrm{m}$ laser are $0.712 \mathrm{~W}$ and $0.361 \mathrm{~W}$, respectively.

\section{Summary}

In summary, we first realize a CW single-frequency tunable $1.08 \mu \mathrm{m}$ laser by utilizing a Nd:CYA crystal as the laser medium, to the best of our knowledge. The maximal output power of the $1 \mathrm{~W}$ CW SLM $1.08 \mu \mathrm{m}$ laser is attained with the slope efficiency of $38.04 \%$. The measured peak-to-peak power stability in $4 \mathrm{~h}$ and beam qualities of the $1.08 \mu \mathrm{m}$ laser are $\pm 0.86 \%$ and $M_{x}^{2}=1.11$, $M_{y}^{2}=1.06$, respectively. Through inserting an IE with thickness of $0.5 \mathrm{~mm}$ into the cavity, the maximal tuning range of $183.71 \mathrm{GHz}$ and continuous tuning range of $60.72 \mathrm{GHz}$ with the free mode-hop of the single-frequency $1.08 \mu \mathrm{m}$ are achieved. The presented CW SLM tunable $1.08 \mu \mathrm{m}$ laser with good 
performance could be applied in atomic physics to precisely match the cesium $6 \mathrm{~S} \rightarrow 7 \mathrm{~S}$ two-photon transition line.

\section{Acknowledgement}

This study was supported by the National Natural Science Foundation of China (Nos. 61975100 and 62027821), Program for the Innovative Talents of High Education Institutions of Shanxi, and Fund for Shanxi "1331 Project" Key Subjects Construction.

\section{References}

1. Y. J. Wang, Y. H. Tian, X. C. Sun, L. Tian, and Y. H. Zheng, "Noise transfer of pump field noise with analysis frequency in a broadband parametric downconversion process," Chin. Opt. Lett. 19, 052703 (2021).

2. Z. Y. Ou, S. F. Pereira, H. J. Kimble, and K. C. Peng, "Realization of the Einstein-Podolsky-Rosen paradox for continuous variables," Phys. Rev. Lett. 68, 3663 (1992).

3. X. L. Su, A. H. Tan, X. J. Jia, J. Zhang, C. D. Xie, and K. C. Peng, "Experimental preparation of quadripartite cluster and $\mathrm{GHz}$ entangled states for continuous variables," Phys. Rev. Lett. 98, 070502 (2007).

4. X. L. Su, Y. P. Zhao, S. H. Hao, X. J. Jia, C. D. Xie, and K. C. Peng, "Experimental preparation of eight-partite cluster state for photonic qumodes," Opt. Lett. 37, 5178 (2012).

5. X. L. Su, S. H. Hao, X. W. Deng, L. Y. Ma, M. H. Wang, X. J. Jia, C. D. Xie, and K. C. Peng, "Gate sequence for continuous variable one-way quantum computation," Nat. Commun. 4, 2828 (2013).

6. X. W. Deng, Y. Xiang, C. X. Tian, G. Adesso, Q. Y. He, Q. H. Gong, X. L. Su, C. D. Xie, and K. C. Peng, "Demonstration of monogamy relations for Einstein-Podolsky-Rosen steering in Gaussian cluster states," Phys. Rev. Lett. 118, 230501 (2017).

7. Y. L. Tian, P. F. Yang, W. Wu, S. K. Li, G. Li, P. F. Zhang, and T. C. Zhang, "Precision measurement of cesium 6S-7S two-photon spectra with single trapped atoms," Jpn. J. Appl. Phys 58, 042002 (2019).

8. D. Z. Li, X. D. Xu, J. Q. Meng, D. H. Zhou, C. T. Xia, F. Wu, and J. Xu, "Diodepumped continuous wave and Q-switched operation of crystal," Opt. Express 18, 18649 (2010).
9. R. V. Horacio and L. M. Thomas, "Nd:CaYAIO lasers emitting at $1.08 \mu \mathrm{m}$," Appl. Phys. Lett. 56, 608 (1990).

10. E. Stephens and L. D. Schearer, "A tunable Nd:CaYAlO 4 laser," Opt. Commun. 90, 79 (1992).

11. H. H. Yu, X. D. Xu, D. Z. Li, Z. P. Wang, and J. Xu, "High-power disordered lasers at $1.08 \mu \mathrm{m}$," Opt. Lett. 35, 2666 (2010).

12. X. H. Fu, Y. Che, and Y. L. Li, "Diode-pumped Nd:CaYAlO ${ }_{4}-\mathrm{KTP}$ intracavity-doubled green laser at $540 \mathrm{~nm}$," Laser Phys. 21, 1021 (2011).

13. Y. S. Zhang, J. Y. Zou, W. X. Zheng, K. Feng, B. Xu, and Z. F. Yu, "Wattlevel continuous-wave intracavity frequency-doubled Pr:YLF-LBO laser at 320 nm," Chin. Opt. Lett. 19, 091406 (2021).

14. K. I. Martin, W. A. Clarkson, and D. C. Hanna, "Self-suppression of axial mode hopping by intracavity second-harmonic generation," Opt. Lett. 22, 375 (1997).

15. S. Greenstein and M. Rosenbluh, "The influence of nonlinear spectral bandwidth on single longitudinal mode intra-cavity second harmonic generation," Opt. Commun. 248, 241 (2005).

16. D. H. Zhou, X. D. Xu, X. Y. Chen, H. M. Zhu, D. Z. Li, J. Q. Di, C. T. Xia, F. Wu, and J. Xu, "CaYAlO 4 crystal growth and spectroscopic properties of $\mathrm{Er}^{3+}$-doped," Phys. Status Solidi A 209, 730 (2012).

17. Z. Zhuo, T. Li, X. M. Li, and H. Z. Ya, "Continuous-wave laser operation of diode-pumped Nd:YVO $/ \mathrm{YVO}_{4}$ composite crystal,” Chin. Opt. Lett. 5, S13 (2007).

18. D. Z. Li, X. D. Xu, H. M. Zhu, X. Y. Chen, W. D. Tan, J. Zhang, D. Y. Tang, J. Ma, F. Wu, C. T. Xia, and J. Xu, "Characterization of laser crystal Yb:CaYAlO ${ }_{4}$," J. Opt. Soc. Am. B 28, 1650 (2011).

19. S. Z. Lv, Z. J. Zhu, Y. Wang, Z. Y. You, J. F. Li, and C. Y. Tu, "Spectroscopic investigations of $\mathrm{Ho}^{3+} / \mathrm{Er}^{3+}: \mathrm{CaYAlO}_{4}$ and $\mathrm{Eu}^{3+} / \mathrm{Er}^{3+}: \mathrm{CaYAlO}_{4}$ crystals for $2.7 \mu \mathrm{m}$ emission," J. Lumin. 144, 117 (2013).

20. P. Loiko, P. Becker, L. Bohaty, C. Liebald, M. Peltz, S. Vernay, D. Rytz, J. M. Serres, X. Mateos, Y. Wang, X. D. Xu, J. Xu, A. Major, A. Baranov, U. Griebner, and V. Petrov, "Sellmeier equations, group velocity dispersion, and thermo-optic dispersion formulas for $\mathrm{CaLnAlO}_{4}(\mathrm{Ln}=\mathrm{Y}, \mathrm{Gd})$ laser host crystals," Opt. Lett. 42, 2275 (2017).

21. P. X. Jin, H. D. Lu, Q. W. Yin, J. Su, and K. C. Peng, "Expanding continuous tuning range of a cw single-frequency laser by combining an intracavity etalon with a nonlinear loss," IEEE J. Sel. Top. Quantum Electron. 42, 16005005 (2017).

22. F. Q. Li, B. Zhao, J. Wei, P. X. Jin, H. D. Lu, and K. C. Peng, "Continuously tunable single-frequency $455 \mathrm{~nm}$ blue laser for high-state excitation transition of cesium," Opt. Lett. 44, 3785 (2019). 